\title{
Data-driven design of bi-selective OSDAs for intergrowth zeolites
}

\author{
Daniel Schwalbe-Koda, ${ }^{\dagger}$ Avelino Corma, ${ }^{\ddagger}$ Yuriy Román-Leshkov, $₫$ Manuel \\ Moliner, ${ }^{\ddagger}$ and Rafael Gómez-Bombarelli*,†
}

$\dagger$ Department of Materials Science and Engineering, Massachusetts Institute of Technology, Cambridge, MA 02139

$\ddagger$ Instituto de Tecnología Química, Universitat Politècnica de València-Consejo Superior de Investigaciones Científicas, Avenida de los Naranjos s/n, 46022 Valencia, Spain. IDepartment of Chemical Engineering, Massachusetts Institute of Technology, Cambridge, MA 02139

E-mail: rafagb@mit.edu

\begin{abstract}
Zeolites are inorganic materials with wide industrial applications due to their topological diversity. Tailoring confinement effects in zeolite pores, for instance by crystallizing intergrown frameworks, can improve their catalytic and transport properties, but controlling zeolite crystallization often relies on heuristics. In this work, we use computational simulations and data mining to design organic structure-directing agents (OSDAs) to favor the synthesis of intergrown zeolites. First, we propose design principles to identify OSDAs which are selective towards both end members of the disordered structure. Then, we mine a database of hundreds of thousands of zeolite-OSDA pairs and downselect OSDA candidates to synthesize known intergrowth zeolites such as CHA/AFX, MTT/TON, and BEC/ISV. The computationally designed OSDAs balance
\end{abstract}


phase competition metrics and shape selectivity towards the frameworks, thus bypassing expensive dual-OSDA approaches typically used in the synthesis of intergrowths. Finally, we propose potential OSDAs to obtain hypothesized disordered frameworks such as AEI/SAV. This work may accelerate zeolite discovery through data-driven synthesis optimization and design.

Zeolites are nanoporous materials with a myriad of applications in catalysis and separations. ${ }^{1,2}$ Thanks to a broad diversity in topology and composition, their shape selectivity can be tuned along with their thermal stability and catalytic activity. ${ }^{3}$ While the complex polymorphism observed in zeolites is key to creating tailored nanoporous materials for targeted applications, it hinders synthesis efforts due to phase competition between different metastable structures. Often, crystallizing a desired framework requires labor-intensive optimization of synthesis conditions to overcome the nucleation and growth of competing phases. Synthesis routes selective towards one framework usually rely on organic structure-directing agents (OSDAs) which template the pore and cage structure of the targeted material. ${ }^{4}$ However, selection of cost-effective OSDAs to crystallize the desired frameworks has historically depended on trial-and-error processes. ${ }^{5}$ While computational approaches have been developed to design OSDAs de novo, ${ }^{6-9}$ these methods often lead to complex OSDAs with low synthetic accessibility or low selectivity towards the targeted framework. ${ }^{10}$

Compared to pure-phase frameworks, intergrown zeolites exhibit a structure that is disordered in at least one dimension. Typically, intergrowths are formed by periodic building units related to each other by symmetry operations such as rotations or translations, ${ }^{11}$ and whose stacking enables them to form a defect-free interface between two topologically distinct domains. Although manually describing such structural faults is possible, ${ }^{11}$ computational methods have enabled enumerating intergrown families such as ABC- $6{ }^{12}$ or simulating diffraction patterns. ${ }^{13}$ More recently, structural descriptors have been proposed to quantify whether two phases are topologically or geometrically compatible to form intergrown structures. $^{14}$ 
As differences in local crystallographic environments affect confinement and transport properties, intergrowth zeolites display catalytic behavior distinct from the pure end-member phases. ${ }^{15,16}$ For example, the silicoaluminophosphate form of the CHA/AEI intergrowth is a competitive catalyst for the methanol-to-olefin (MTO) reaction compared to the commercial SAPO-34 catalyst. ${ }^{17,18}$ However, the challenges of selective zeolite synthesis are exacerbated in the case of intergrowths. Traditional routes for synthesizing the high-silica form of CHA/AEI require the combination of two specific OSDAs for CHA and AEI, ${ }^{19}$ which increases the synthesis complexity and cost. In addition, tuning the synthesis conditions to favor the formation of the desired intergrowth requires labor-intensive experimentation. Nevertheless, this dual-OSDA approach has been the standard for crystallizing several intergrown zeolites, ${ }^{20}$ despite being limited by: (i) the need to design two equally-selective OSDAs, (ii) the heuristic optimization of the synthesis conditions to avoid the collapse to a single phase, and (iii) avoiding formation of extraneous competing phases.

Recently, we have performed high-throughput simulations to quantify phase competition in zeolites. ${ }^{10,21,22}$ The large number of simulated zeolite-OSDA pairs allowed rationalizing historical synthesis outcomes from the literature, explaining accessibility windows, and aluminum distributions in single zeolite frameworks as a function of OSDA shape and charge distribution. Furthermore, the data was used to control the synthesis of an aluminosilicate CHA/AEI intergrowth with an OSDA with dual selectivity. By designing OSDAs with shapes and binding energies competitive towards the two frameworks forming the intergrowth, we have demonstrated that this disordered structure can be crystallized in its high-silica form. ${ }^{10}$ In this work, we extend these design principles towards several other intergrown zeolites by combining simulations and data mining. Starting from a dataset of hundreds of thousands of zeolite-OSDA pairs, we singled out potential bi-selective OSDAs for the synthesis of both known and hypothesized intergrowths. To demonstrate the applicability of the method towards small, medium, and large-pore zeolites, we designed potential bi-selective OSDAs for the CHA/AFX, MTT/TON, and BEC/ISV intergrowths. Finally, the strategy is extended 
to several known frameworks, and one example of a hypothesized intergrowth. This work is expected to accelerate the discovery of intergrowth zeolites through theory and simulations.

This new computational strategy for designing an intergrowth zeolite is shown in Fig. 1. OSDAs known to be selective towards pure phase zeolites are used as references for the shape, such as N-ethyl-N-methyl-2,2,6,6-tetramethylpiperidinium for $\mathrm{AEI}^{23}$ and N,N,Ntrimethyladamantammonium for $\mathrm{CHA}^{24}$ (Fig. 1a). Then, new OSDAs for the intergrowth can be designed using phase competition and shape metrics. Binding metrics built upon binding energies were calculated for OSDAs retrieved from the literature and for chemically modified ones. ${ }^{10,25}$ In particular, we define the competition energy by comparing the binding energies of one OSDA towards all calculated frameworks, and adopting the second-best zeolite-OSDA pair as the energy reference. This quantifies how strong are the phase competition effects for a given OSDA, with more negative values indicating less competition. Moreover, the shape of an OSDA is quantified by first projecting nuclear coordinates of the molecule into a two-dimensional space using a principal component analysis, and later measuring the two axes describing the molecule (see Supporting Information). ${ }^{10}$ When designing bi-selective OSDAs for intergrowth systems, we postulate that an ideal bi-selective OSDA should: (i) be an equally strong binder towards both phases forming the intergrowth, (ii) be a weak binder towards all other competing phases (Fig. 1b), and (iii) have a geometry that is in-between the reference OSDAs in the shape space, thus favoring the crystallization of both structures (Fig. 1c). ${ }^{10}$

Beyond the previously demonstrated CHA/AEI intergrowth, we discuss the application of this method to many other intergrown zeolites of practical interest. For example, the highsilica CHA/AFX zeolite is used in applications such as MTO reaction, or selective catalytic reduction of $\mathrm{NO}_{\mathrm{x}}\left(\mathrm{NO}_{\mathrm{x}}-\mathrm{SCR}\right)$, but is typically synthesized using a mixture of OSDAs. ${ }^{20}$ Although both zeolites are ABC-6 structures and have cages with similar diameters, the aft cage in AFX zeolite is $40 \%$ longer than the cha cage. This discrepancy in size has led AFX to be synthesized with longer OSDAs, including diquaternary ammonium molecules such 


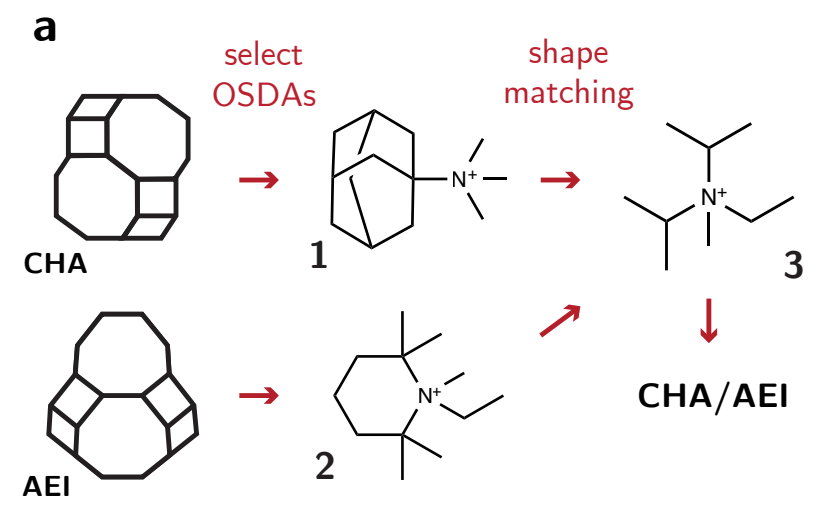

b

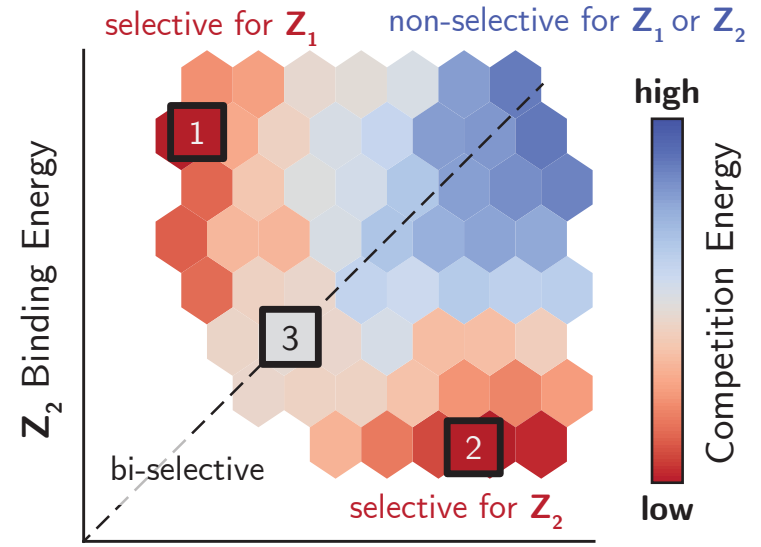

C

$\mathbf{Z}_{1}$ Binding Energy

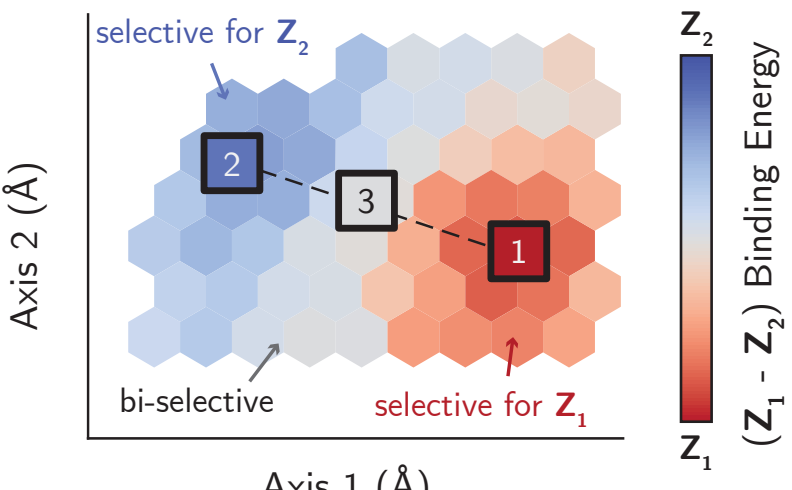

Axis $1(\AA)$

Figure 1: a, Diagram of the method. Starting from known, selective OSDAs for the synthesis of pure-phase components, a shape interpolation is performed to locate a bi-selective OSDA. b. Schematic of predicted OSDA selectivity towards the pure zeolites and the intergrowth. Along with the binding energies, bi-selective OSDAs should also satisfy c, shape matching requirements. Often, binding energy metrics give rise to selectivity domains in the shape space. 
as 1,4-bis(1-azoniabicyclo[2.2.2] octane)butyl, ${ }^{26}$ or monocationic OSDAs such as 1,3-bis(1adamantyl)imidazolium. ${ }^{27}$

Figure 2 shows the proposed OSDAs for a CHA/AFX intergrowth based on the biselectivity design principles. As the gme cage in the AFX framework is not occupied by an OSDA, but may be stabilized by an inorganic structure-directing agent, the binding energy per $\mathrm{SiO}_{2}$ alone, as is traditionally normalized, does not determine the likelihood of crystallizing this framework. ${ }^{10}$ Quantifying phase competition effects of both inorganic and organic structure-directing agents at the same could provide additional insights on these phase boundaries, but simulations with these effects are overly expensive. Instead, the proposed OSDAs can also be compared with respect to their binding energy normalized per OSDA (Table S1 in the Supporting Information), which has been proven useful to describe structure-direction in cage-based zeolites. ${ }^{10}$ All computer-designed OSDAs in Fig. 2b exhibit intermediate binding energies both for $\mathrm{CHA}$ and AFX. In addition, the templates are longer than TMAda, as required to favor the formation of the AFX framework in addition to the cha cage. Interestingly, most of the proposed OSDAs may crystallize the aft cage by promoting intermolecular $\pi-\pi$ stacking, which enables two molecules to fit in a single aft cage (Fig. S1 in the Supporting Information). On the other hand, flexible OSDAs may favor the crystallization of the cha cage by adopting a folded conformation, or the aft cage by adopting the longer conformation (Fig. S2).

The MTT and TON zeolites also form intergrowths of practical interest. Both zeolites have one-dimensional medium-sized pores and can be synthesized in the aluminosilicate form. The slight differences in pore sizes of MTT and TON could lead to membranes with high shape selectivity, as has been observed for the MEL/MFI case. ${ }^{28}$ However, the synthesis of an MTT/TON intergrowth is typically performed by using the dual-OSDA approach, hindering the control of phase fraction. ${ }^{29}$ Figure 3 shows monocationic OSDAs proposed to enable the control of the MTT/TON intergrowth. Differently from Fig. 2a, MTT or TON are not the best hosts for most OSDAs based on the phase competition metrics (Fig. 3c). 
a

b<smiles>CCC[N+]12CCC(CC1)CC2</smiles><smiles>C[N+]1(C)CC2CC=CCC2C1</smiles><smiles>CCC[N+]1(C)CCCC1</smiles><smiles></smiles><smiles></smiles><smiles>C[C@H]1Cc2ccccc2C[N+]1(C)C</smiles><smiles>CC1Cc2c[13c]ccc2[N+]1(C)C</smiles>

C

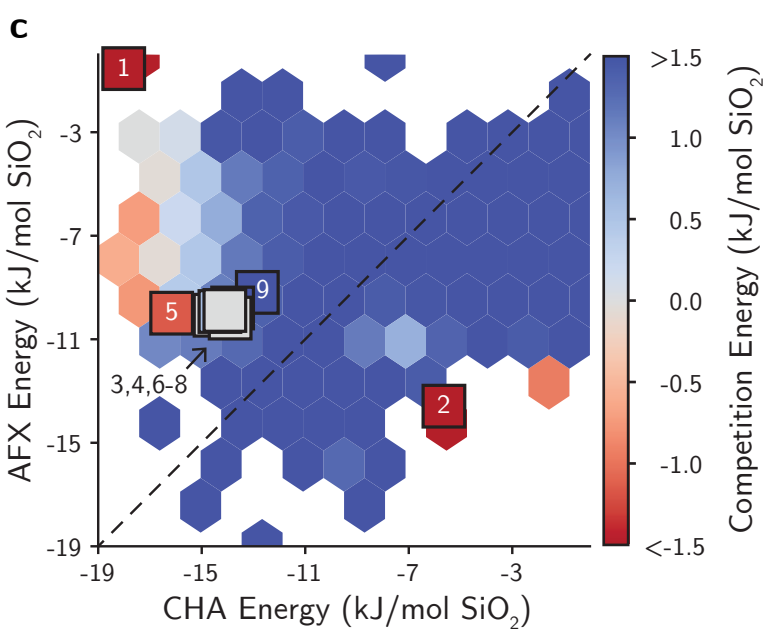

d

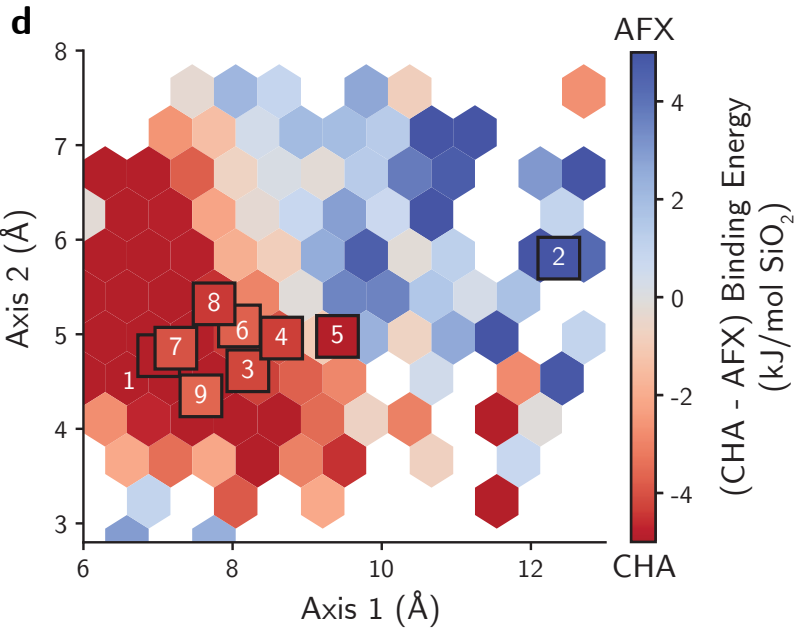

Figure 2: a, Reference OSDAs for the synthesis of CHA and AFX. b, Proposed OSDAs for the synthesis of CHA/AFX intergrowth. c, Phase competition metrics and $\mathbf{d}$, shape matching between $\mathrm{CHA}$ and AFX. 
However, fine-tuning the synthesis conditions may direct the formation of these zeolites and the corresponding intergrowth. Although synthesis parameters cannot be selected purely through simulations, past literature outcomes and machine learning models trained on them may guide the exploration of synthesis routes. ${ }^{25,30}$ Interestingly, since MTT has a slightly smaller unit cell than TON, variations of binding energy with respect to shape depend on the commensurability of the OSDA within the framework (Fig. 3d). At a value of $4 \AA$ for Axis 1, OSDAs are more favorable towards MTT than TON. As this value increases, the color shifts towards blue, indicating that slightly longer OSDAs favor TON over MTT. Since the zeolites are simulated with supercells, the pattern starts to repeat at a value of $8 \AA$ for Axis 1 . This suggests that an MTT/TON intergrowth could be controlled by varying the length of the molecule, in addition to its binding energy. The examples of OSDAs shown in Fig. 3b exhibit a variety of lengths, and are likely to direct the formation of the MTT/TON intergrowth.

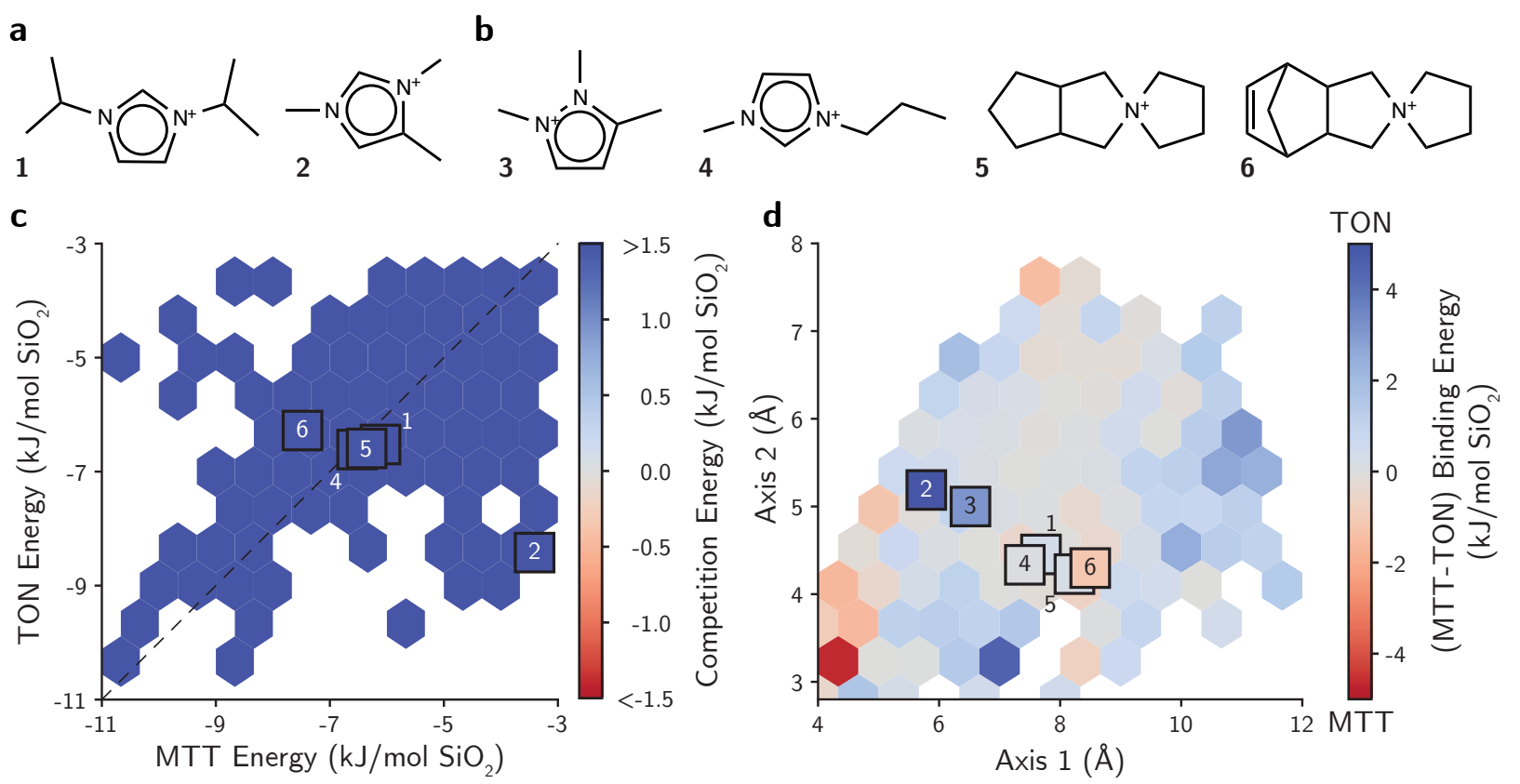

Figure 3: a, Reference OSDAs for the synthesis of MTT and TON. b, Proposed OSDAs for the synthesis of MTT/TON intergrowth. c, Phase competition metrics and $\mathbf{d}$, shape matching between MTT and TON.

Finally, we show that the method can also be applied to large-pore, three dimensional 
zeolites. One example is the BEC/ISV intergrowth, which is often only obtained in the presence of germanium or fluoride in the synthesis media. ${ }^{31}$ Despite the challenge usually associated to predicting OSDAs for zeolites with intersecting pores, the design principles successfully recover the literature results for phase selectivity in BEC and ISV. Figure 4 shows the proposed OSDAs for the synthesis of this intergrown structure. Reference OSDAs for the selective synthesis of BEC are indeed more favorable towards this framework compared to ISV (Fig. 4a,c). Furthermore, the red area towards OSDAs with longer Axis 1 in Fig. 4d recover the fact that longer OSDAs favor BEC zeolite, since the unit cells of BEC and ISV have very similar $a$ and $b$ lattice parameters, but ISV has a $c$ lattice parameter $1 \%$ smaller than the $c$ in the BEC unit cell. Similarly to the MTT/TON example, this indicates that the commensurability between the OSDA and the zeolite is a useful parameter when predicting phase selectivity. From these insights and the results from Fig. 4d, we suggest that OSDAs with an Axis 1 between 9 and $10 \AA$ lie in the phase boundary between BEC and ISV, and could be used to direct a zeolite intergrowth.

Since this rationale can be extended to any pair of zeolites that are individually templated by OSDAs, bi-selective OSDAs leading to other intergrown structures can be predicted. In Figs. S3-10 in the Supporting Information, we show the applicability of this method towards many other intergrowths, including known disordered structures such as SBS/SBT, FAU/EMT, MEL/MFI, ITH/ITR, RTH/ITE, and ERI/OFF. In cases such as MEL/MFI or RTH/ITE (Figs. S3,4), the difficulty in distinguishing between phase boundaries may explain why these phases are typically found as intergrowths even when a single OSDA is used. As the shapes of the cavities are very similar, bi-selectivity is more easily achieved compared to other intergrowths. In other cases such as FAU/EMT, ITH/ITR, ERI/OFF, AFS/BPH (Figs. S5-8), the shape boundaries are more subtle, and often dominated by one of the end members. This suggests that balancing phase competition between the polymorphs could require tuning other synthesis conditions in addition to the OSDA. This is also the case of SBS/SBT (Fig. S9), whose intergrowth can be tuned beyond the use of bi-selective 


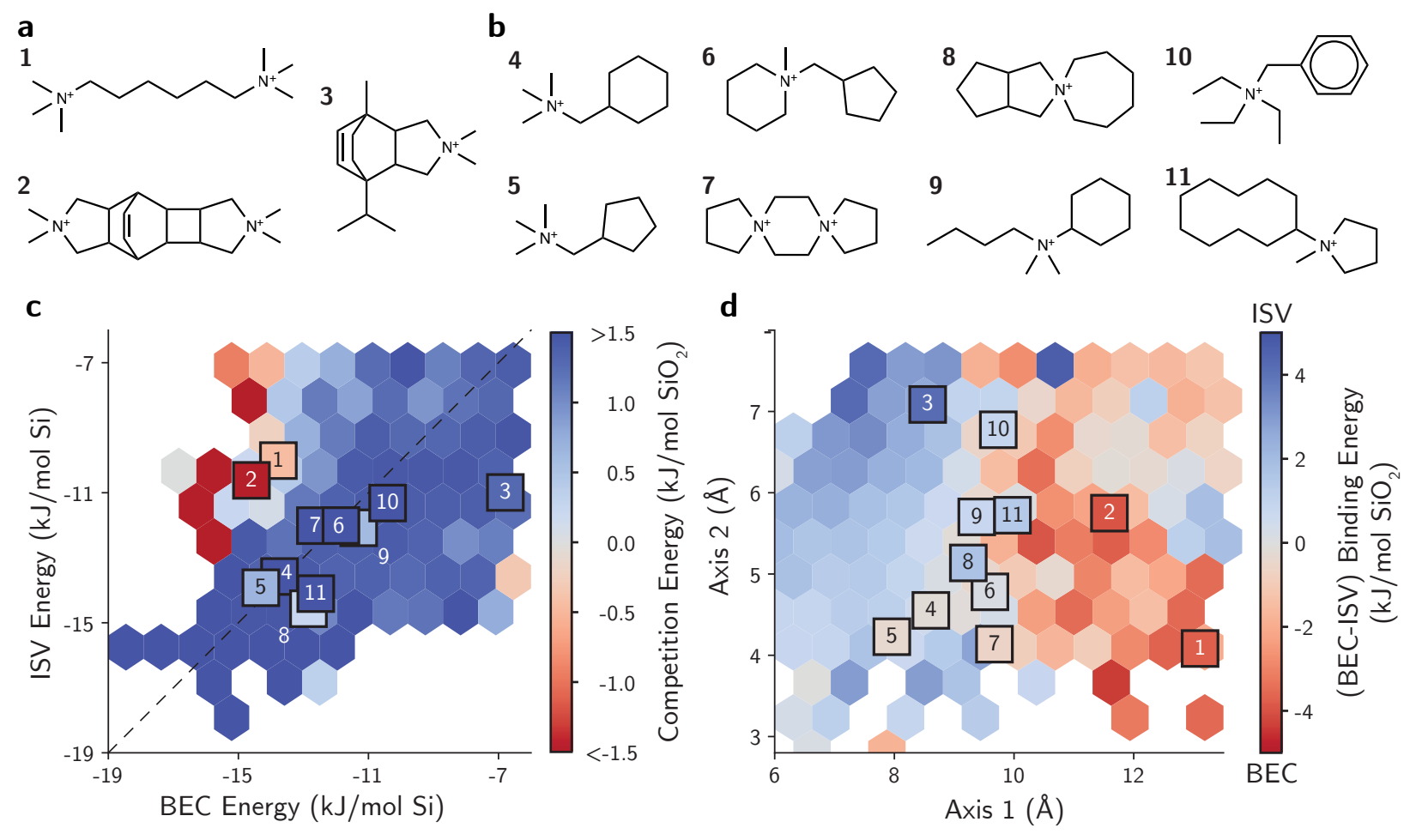

Figure 4: a, Reference OSDAs for the synthesis of BEC and ISV. b, Proposed OSDAs for the synthesis of BEC/ISV intergrowth. $\mathbf{c}$, Phase competition metrics and $\mathbf{d}$, shape matching between BEC and ISV. 
OSDAs by selecting inorganic structure-directing agents that cooperate to crystallize the

disordered structure. ${ }^{32}$ Finally, OSDAs for realizing the hypothetical intergrowth AEI/SAV (Fig. S10) is proposed. Whereas SAV is typically synthesized as a zeotype, synthesizing an aluminosilicate SAV framework intergrown with SSZ-39 (AEI framework) may be interesting for catalytic applications such as $\mathrm{NO}_{\mathrm{x}}$-SCR beyond pure AEI or CHA/AEI intergrowths.

In summary, we combined simulations, data mining, and design principles to propose bi-selective OSDAs for intergrowth zeolites. By visualizing the OSDAs according to their phase competition and shape matching metrics, we designed promising bi-selective candidates for cost-efficient synthesis of intergrowth zeolites. The method was demonstrated for small-, medium-, and large-pore zeolites, and extended towards several known intergrowths. Furthermore, we proposed OSDAs that could realize a hypothetical intergrowth. This work is expected to accelerate discovery of intergrown zeolites using computation.

\section{Supporting Information Available}

Computational methods, visualizations and comparisons of binding modes/metrics for proposed OSDAs in CHA/AFX zeolites, and proposed OSDAs for 8 intergrowth zeolite pairs.

\section{Acknowledgement}

This work was supported by the MIT Energy Initiative (MITEI) and MIT International Science and Technology Initiatives (MISTI) Seed Funds. D.S.-K. was additionally funded by the MIT Energy Fellowship. Computer calculations were executed at the Massachusetts Green High-Performance Computing Center with support from MIT Research Computing. 


\section{References}

(1) Vermeiren, W.; Gilson, J.-P. Impact of Zeolites on the Petroleum and Petrochemical Industry. Topics in Catalysis 2009, 52, 1131-1161.

(2) Davis, M. E. Ordered porous materials for emerging applications. Nature 2002, 417, $813-821$.

(3) Li, Y.; Li, L.; Yu, J. Applications of Zeolites in Sustainable Chemistry. Chem 2017, 3, 928-949.

(4) Moliner, M.; Rey, F.; Corma, A. Towards the Rational Design of Efficient Organic Structure-Directing Agents for Zeolite Synthesis. Angewandte Chemie International Edition 2013, 52, 13880-13889.

(5) Gallego, E. M.; Portilla, M. T.; Paris, C.; León-Escamilla, A.; Boronat, M.; Moliner, M.; Corma, A. "Ab initio" synthesis of zeolites for preestablished catalytic reactions. Science 2017, 355, 1051-1054.

(6) Lewis, D. W.; Willock, D. J.; Catlow, C. R. A.; Thomas, J. M.; Hutchings, G. J. De novo design of structure-directing agents for the synthesis of microporous solids. Nature 1996, 382, 604-606.

(7) Sastre, G.; Cantin, A.; Diaz-Cabañas, M. J.; Corma, A. Searching Organic Structure Directing Agents for the Synthesis of Specific Zeolitic Structures: An Experimentally Tested Computational Study. Chemistry of Materials 2005, 17, 545-552.

(8) Pophale, R.; Daeyaert, F.; Deem, M. W. Computational prediction of chemically synthesizable organic structure directing agents for zeolites. Journal of Materials Chemistry A 2013, 1, 6750-6760.

(9) Muraoka, K.; Chaikittisilp, W.; Okubo, T. Multi-objective de novo molecular design 
of organic structure-directing agents for zeolites using nature-inspired ant colony optimization. Chemical Science 2020, 11, 8214-8223.

(10) Schwalbe-Koda, D.; Kwon, S.; Paris, C.; Bello-Jurado, E.; Jensen, Z.; Olivett, E.; Willhammar, T.; Corma, A.; Román-Leshkov, Y.; Moliner, M.; Gómez-Bombarelli, R. A priori control of zeolite phase competition and intergrowth with high-throughput simulations. Science 2021, abh3350.

(11) Ch. Baerlocher and L.B. McCusker, Database of Zeolite Structures. http://www.izastructure.org/databases/2021,

(12) Li, H.; Leung, K. S.; Wong, M. H.; Ballester, P. J. Correcting the impact of docking pose generation error on the prediction of binding affinity. BMC Bioinformatics 2015, 8623, 231-241.

(13) Treacy, M. M.; Newsam, J. M.; Deem, M. W. A general recursion method for calculating diffracted intensities from crystals containing planar faults. Proceedings of the Royal Society of London. Series A: Mathematical and Physical Sciences 1991, 433, 499-520.

(14) Schwalbe-Koda, D.; Jensen, Z.; Olivetti, E.; Gómez-Bombarelli, R. Graph similarity drives zeolite diffusionless transformations and intergrowth. Nature Materials 2019, $18,1177-1181$.

(15) Willhammar, T.; Sun, J.; Wan, W.; Oleynikov, P.; Zhang, D.; Zou, X.; Moliner, M.; Gonzalez, J.; Martínez, C.; Rey, F.; Corma, A. Structure and catalytic properties of the most complex intergrown zeolite ITQ-39 determined by electron crystallography. Nature Chemistry 2012, 4, 188-194.

(16) Bates, J. S.; Bukowski, B. C.; Harris, J. W.; Greeley, J.; Gounder, R. Distinct Catalytic Reactivity of Sn Substituted in Framework Locations and at Defect Grain Boundaries in Sn-Zeolites. ACS Catalysis 2019, 9, 6146-6168. 
(17) Guo, L.; Zhu, W.; Miao, P.; Li, F.; Guo, Z.; Sun, Q. Intergrowth Silicoaluminophosphate Molecular Sieves Synthesized and Their Catalytic Performances for Methanol to Olefins Reaction. Industrial 65 Engineering Chemistry Research 2018, 57, 10398-10402.

(18) Smith, R. L.; Svelle, S.; del Campo, P.; Fuglerud, T.; Arstad, B.; Lind, A.; Chavan, S.; Attfield, M. P.; Akporiaye, D.; Anderson, M. W. CHA/AEI intergrowth materials as catalysts for the Methanol-to-Olefins process. Applied Catalysis A: General 2015, 505, 1-7.

(19) Cao, G.; Mertens, M. M.; Strohmaier, K. G.; Hall, R. B.; Colle, T. H.; Afeworki, M.; Bons, A. J.; Mortier, W. J.; Kliewer, C.; Li, H.; Guram, A. S.; Saxton, R. J.; Muraoka, M. T.; Yoder, J. C. Chabazite-containing molecular sieve, its synthesis and its use in the conversion of oxygenates to olefins. US Patent 7,094,389 B2 2006,

(20) Naraki, Y.; Ariga, K.; Nakamura, K.; Okushita, K.; Sano, T. ZTS-1 and ZTS-2: Novel intergrowth zeolites with AFX/CHA structure. Microporous and Mesoporous Materials 2017, 254, 160-169.

(21) Schwalbe-Koda, D.; Gomez-Bombarelli, R. Supramolecular Recognition in Crystalline Nanocavities Through Monte Carlo and Voronoi Network Algorithms. Journal of Physical Chemistry C 2021, 125, 3009-3017.

(22) Schwalbe-Koda, D.; Gomez-Bombarelli, R. Benchmarking binding energy calculations for organic structure-directing agents in pure-silica zeolites. Journal of Chemical Physics 2021, 154, 174109.

(23) Schmidt, J. E.; Deem, M. W.; Lew, C.; Davis, T. M. Computationally-Guided Synthesis of the 8-Ring Zeolite AEI. Topics in Catalysis 2015, 58, 410-415.

(24) Zones, S. I. Conversion of faujasites to high-silica chabazite SSZ-13 in the presence of N,N,N-trimethyl-1-adamantammonium iodide. Journal of the Chemical Society, Faraday Transactions 1991, 87, 3709-3716. 
(25) Jensen, Z.; Kwon, S.; Schwalbe-Koda, D.; Paris, C.; Gómez-Bombarelli, R.; RománLeshkov, Y.; Corma, A.; Moliner, M.; Olivetti, E. A. Discovering Relationships between OSDAs and Zeolites through Data Mining and Generative Neural Networks. ACS Central Science 2021, 7, 858-867.

(26) Zones, S. Zeolite SSZ-13 and its method of preparation. US Patent 4,544,538 1985,

(27) Archer, R. H.; Zones, S. I.; Davis, M. E. Imidazolium structure directing agents in zeolite synthesis: Exploring guest/host relationships in the synthesis of SSZ-70. Microporous and Mesoporous Materials 2010, 130, 255-265.

(28) Kumar, P.; Kim, D. W.; Rangnekar, N.; Xu, H.; Fetisov, E. O.; Ghosh, S.; Zhang, H.; Xiao, Q.; Shete, M.; Siepmann, J. I.; Dumitrica, T.; McCool, B.; Tsapatsis, M.; Mkhoyan, K. A. One-dimensional intergrowths in two-dimensional zeolite nanosheets and their effect on ultra-selective transport. Nature materials 2020, 19, 443-449.

(29) Wang, B.; Tian, Z.; Li, P.; Wang, L.; Xu, Y.; Qu, W.; Ma, H.; Xu, Z.; Lin, L. Synthesis of ZSM-23/ZSM-22 intergrowth zeolite with a novel dual-template strategy. Materials Research Bulletin 2009, 44, 2258-2261.

(30) Jensen, Z.; Kim, E.; Kwon, S.; Gani, T. Z. H.; Román-Leshkov, Y.; Moliner, M.; Corma, A.; Olivetti, E. A Machine Learning Approach to Zeolite Synthesis Enabled by Automatic Literature Data Extraction. ACS Central Science 2019, 5, 892-899.

(31) Villaescusa, L. A.; Díaz, I.; Barrett, P. A.; Nair, S.; LLoris-Cormano, J. M.; MartínezMañez, R.; Tsapatsis, M.; Liu, Z.; Terasaki, O.; Camblor, M. A. Pure Silica Large Pore Zeolite ITQ-7: Synthetic Strategies, Structure-Directing Effects, and Control and Nature of Structural Disorder. Chemistry of Materials 2007, 19, 1601-1612.

(32) Lee, H.; Shin, J.; Lee, K.; Choi, H. J.; Mayoral, A.; Kang, N. Y.; Hong, S. B. Synthesis of thermally stable SBT and SBS/SBT intergrowth zeolites. Science 2021, 373, 104 $\mathrm{LP}-107$. 